\title{
Two Editors, A Common Vision, and Several Changes
}

\author{
Craig Bennell • Brent Snook
}

Published online: 10 November 2010

(C) Springer Science+Business Media, LLC 2011

It is with great excitement and much honor that we take on the role of co-editing the Journal of Police and Criminal Psychology (JPCP), the official journal of the Society for Police and Criminal Psychology. We are excited because this co-editorship represents a new challenge for us and provides us with the opportunity to help shape the direction of the two major fields represented by this journal. We are honored because we have been asked to edit a journal that represents the opinions of all those remarkable people who conduct research in the fields of police and criminal psychology, many of whom are long-standing members of the Society.

As is customary when assuming an editorship, we would like to discuss our vision of where we see JPCP going in the future and highlight some changes that can be expected during our term as co-editors. Before we do this, however, we would like to acknowledge the great job done by Alan Thompson during his term as editor and thank him and the rest of the Springer team for providing us with much needed guidance during the transition period. Thanks to these individuals, we have been able to get on top of things fairly quickly.

With respect to our vision for the future, we have discussed this at length with each other, with Alan, and with Welmoed Spahr, our trusted publisher. Above all else, our primary goals with the journal will be to increase its visibility and to continually improve its

C. Bennell $(\bowtie)$

Carleton University,

Ottawa, ON, Canada

e-mail: craig_bennell@carleton.ca

B. Snook

Memorial University of Newfoundland,

St. John's, NL, Canada quality. Significant advances in this direction have already been made, thanks to the hard work of Alan and Welmoed. Most notably, the journal is now being indexed with $P$ sycINFO, which means that articles in the journal will be easier to find and easier to cite. Given the growing importance of citation counts for tenure and promotion decisions, we are sure that this recent change will attract researchers that did not previously consider publishing in the journal.

Another clear way to increase the visibility and quality of the journal is to market it to a wider audience. This is something we have already begun. Notices have been sent to colleagues from around the world encouraging them to think about this journal as an outlet for their research. Similar messages have been included in newsletters and newsgroups belonging to brother and sister organizations. Face-to-face advertising has been done at workshops and conferences. We are confident that these efforts will make the journal more visible on the international stage and increase the number of manuscript submissions. Such marketing efforts should also help to ensure that the journal continues to publish high-quality pieces of research.

In our view, one of the most important things that we must do to ensure that authors think seriously about JPCP as an outlet for their research is to continue paying close attention to turnaround times; that is, the time it takes between submitting a manuscript and receiving an editorial decision. For all sorts of reasons, a short turnaround time is desirable and it is critical if we are to be a competitive journal. Compared to many journals, JPCP has always had a relatively short turnaround time, but we are committed to improving this further. As discussed below, this will be accomplished in large part by expanding the number of reviewers used by the journal, by making sure that 
reviewers are committed to conducting high-quality reviews in a reasonable timeframe, and by staying on top of reviewers to ensure that deadlines are met. We are committed to having a maximum turnaround time of 3 months to get back an initial editorial decision, though 2 months is our personal target.

On a related note, the overall time between submission and publication in JPCP is also shortened by the fact that all articles that are accepted for publication will appear on Springer's "Online First", a digital library containing the copyedited articles that will eventually appear in print. All articles in this database can be located via article searches, cited by other researchers, and listed on your CV.

Finally, in terms of our vision for the journal, we plan to continue the long practice of encouraging submissions from a diverse range of researchers, including academics, mental health professionals, and police practitioners of all types (including officers, analysts, administrators, etc.). In addition, we will continue to seek manuscript submissions that relate to basic and applied research because this journal is an outlet for fundamental research that moves criminal justice knowledge forward and for applied research that relates to criminal justice practices, particularly in the policing domain. Our overarching goal is to publish research that is well written and is of high-quality, especially innovative research that has the potential to have a substantial impact on how we think about police and criminal psychology.

With respect to changes that might be expected during our editorship, we would like to discuss five specific points.

The first and most obvious change that readers will notice is that the journal will now be edited by two individuals rather than one. Although this is a direction the journal has never taken, we are completely confident that having co-editors will not cause any problems for the smooth running of the journal. Having collaborated together for a very long time, we have an excellent working relationship with each other and share the same views on where the fields of police and criminal psychology should go. The fact that the journal is now edited by co-editors should also not create any additional confusion for submitting authors, though we would like to briefly inform readers of the procedure that will be followed with manuscript submissions.

As has been the case for some time, authors will submit their manuscripts to the journal through the Springer portal, which we will use to administer the entire review process. At that time, Springer will assign each manuscript (in an alternating fashion) to one of us. From that point on, the assigned editor will take over all responsibilities for handling internal reviews, selecting external reviewers, and making editorial decisions. From start to finish, submitting authors will only deal with one editor and, for all intents and purposes, will not be aware that there are actually two of us at the helm. If people have questions or comments about the journal, they can contact either one of us.

As mentioned above, a second change that has already started to take place is that we are expanding the database of reviewers. It has been the case for some time that a relatively small group of individuals have served as the primary reviewers for the journal, many whom belong to the Society. While these individuals have done a superb job, we have also been informed that many of these reviewers may have been overworked. This is not as it should be. The expansion of the reviewer database will allow us to access colleagues who can provide very detailed, high-quality reviews (due to their specific subject matter expertise) in a relatively short timeframe (by not overburdening any single reviewer).

Thirdly, there will be changes made to the composition of the editorial board. For the next year, we do not intend to change the board in any drastic way, preferring instead to rely on the expertise of those individuals who currently serve on the board. However, over time, we plan to expand the board to include individuals working in areas that are not well represented by current board members. Additions to the board will be made in the standard way-reviewers who have shown a consistently high level of commitment to the journal by submitting quality reviews in a timely fashion will be given high priority, especially if they possess knowledge in an area that is lacking on the board.

Fourthly, we expect that there might be some changes to the structure of the journal. For example, there is some interest on Springer's part to increase the size of the journal, and potentially even move towards a quarterly publication schedule. Although it is still too early for us to know whether a quarterly schedule is feasible, especially given our primary concern of maintaining the quality of the articles that appear in print, we will strive to increase the size of the journal so that more articles are included in each issue.

The final change we would like to see relates to the breadth of the articles published in JPCP. The mandate of the journal is quite broad, covering the full range of topics in the fields of police and criminal psychology. However, in practice, the majority of journal pages are dedicated to research in the area of police psychology. While we would never discourage authors from submitting high-quality police psychology research, we are going to increase our efforts to market the journal to our criminal psychology colleagues. This research can clearly benefit the journal and inform police practice.

Before we sign off, we would like to thank the Society and all of its members (especially the Execu- 
tive) for placing their faith in us. We both realize the value of this journal for the Society and for the fields of police and criminal psychology more generally, and we will do our very best during our term as co-editors. We look forward to meeting the readers of this journal and its contributors in the future. If we happen to be at the same conference or meeting, please do come up to us to talk about the journal, to make suggestions on how we might improve it, or to swing publication ideas by us. If we do not meet you in person, you can always email us at craig_bennell@carleton.ca or bsnook@play. psych.mun.ca. 